\title{
PRECONCENTRATION AND DETERMINATION OF SHORT CHAIN MERCAPTANS IN THE PRODUCED WATER BY DISPERSIVE LIQUID-LIQUID MICROEXTRACTION AND GAS CHROMATOGRAPHY
}

\author{
BAHRAM MOKHTARI ${ }^{1,2 *}$, NASSER DALALI', KOBRA POURABDOLLAH ${ }^{l}$ \\ ${ }^{I}$ Razi Chemistry Research Center (RCRC), Shahreza Branch, Islamic Azad University, Shahreza, Iran. \\ ${ }^{2}$ Phase Separation \& FIA Lab, Department of Chemistry, Faculty of Science, Zanjan University, Zanjan 45195-313, Iran
}

(Received: November 24, 011 - Accepted: August 26, 2012)

\begin{abstract}
This paper describes the preconcentration of short chain n-alkyl $\mathrm{C}_{1}-\mathrm{C}_{6}$ mercaptans in the produced water using the dispersive liquid-liquid microextraction. In the present experiments, $0.8 \mathrm{~mL}$ dispersive solvent (acetonitrile) containing $10.0 \mu \mathrm{L}$ extraction solvent (carbon tetrachloride) was rapidly injected into the samples and followed by centrifuging and direct injection into the gas chromatograph equipped with flame ionization detector. According to the results, dispersive liquid-liquid microextraction gas chromatography flame-ionization detector was successfully used for determination of short chain $\mathrm{n}$-alkyl $\mathrm{C}_{1}-\mathrm{C}_{6}$ mercaptans. The parameters affecting the extraction efficiency were evaluated and optimized. Under the optimum conditions, the figures of merits for six short chain $\mathrm{n}$-alkyl $\mathrm{C}_{1}-\mathrm{C}_{6}$ mercaptans were determined to be 4.0-100.0 $\mathrm{ng} / \mathrm{L}(\mathrm{n}=6, \mathrm{RSD}=6.8 \%), 6.0-80.0 \mathrm{ng} / \mathrm{L}(\mathrm{n}=6, \mathrm{RSD}=11.0 \%), 2.0-90.0 \mathrm{ng} / \mathrm{L}(\mathrm{n}=6, \mathrm{RSD}=2.6 \%), 5.0-120.0 \mathrm{ng} / \mathrm{L}$ $(\mathrm{n}=6, \mathrm{RSD}=4.4 \%), 3.0-80.0 \mathrm{ng} / \mathrm{L}(\mathrm{n}=6, \mathrm{RSD}=9.2 \%)$ and $2.0-110.0 \mathrm{ng} / \mathrm{L}(\mathrm{n}=6, \mathrm{RSD}=6.5 \%)$, respectively.
\end{abstract}

Keywords: Mercaptan; Microextraction; Gas Chromatography; Flame-Ionization Detector.

\section{INTRODUCTION}

Liquid-liquid extraction (LLE) is gradually substituted by other methods for its disadvantage of large consumption of hazardous organic solvents, time-consuming, emulsification and loss of target analytes. Hence, many microextraction methods that involved no or small amount of solvents were developed over the past decade. Among them, liquid-phase microextraction (LPME) achieved extensive attention as a novel sample pretreatment technique owing to its advantages such as simple operation, rapidity, low cost, miniaturization, low consumption of sample volume and extraction solvent. Recently, dispersive liquid-phase microextraction (DLLME) [1] has been developed as a novel and successful mode of LPME. Compared with the conventional LPME methods, DLLME avoids the volatilization and loss of extractants and provides possibility for the enhancement of sensitivity and simplification of the extraction procedure. The other outstanding advantages are its rapidity and inexpensive.

Produced water is a by-product of the production of oil and gas hydrocarbons from underground reservoirs. Water is naturally present in the oil reservoirs, is a by-product of oil production and admixes as a liquid with the oil. In the first stages of processing of the production platforms, the water component is decanted from the oil, and has generally been discharged to the sea.

Witter and Jones [2] determined inorganic sulfur species in produced water of offshore oils using multiple analytical techniques and compared them. Concerning the organic sulfur containing compounds in the produced water, new methods base upon the molecular recognition are presented and the results are reported. In the rest of last researches, Witter and Jones [3] determined the organosulfur compounds in produced waters using gas chromatography/mass spectrometry. However, the mercaptans were not analyzed there.

Chromatographic methods have been used for quantitative and sensitive determination of mercaptans in air and solutions [4-6]. Wang et al. [7] determined the mercaptans in garlic and yellow and green onion and by means of headspace solid-phase microextraction (HS-SPME) and $\mathbf{N}$-phenylmaleimide as an in-fiber derivatization reagent. Similarly, Rodríguez-Bencomo et al. [8], Mestres et al. [9] and Herszage et al. [10] determined some volatile mercaptans in white, rose and red wines.

Li et al. [11] have reviewed the characteristics of common sulfur odorants and organic sulfur compounds in gas chromatography analysis. They discussed the selection roles of detector, chromatographic column, preconcentration method, and prospects of analytical testing techniques for volatile organic sulfur compounds in aqueous samples.

In this work, dispersive liquid-liquid microextraction was used for determination of short chain n-alkyl $\mathrm{C}_{1}-\mathrm{C}_{6}$ mercaptans in the samples of produced water. The figures of merit for this method reveal the selective and sensitive preconcentration of selected amines in the produced water. Figure 1 illustrates the chemical structure of tested mercaptans.

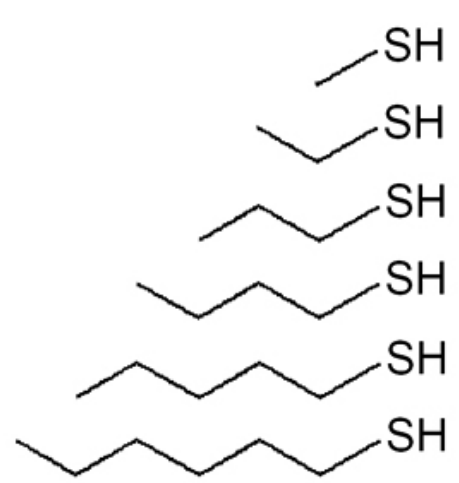

Figure 1. The chemical structure of short chain n-alkyl $\mathrm{C}_{1}-\mathrm{C}_{6}$ mercaptans.

\section{EXPERIMENTAL}

\subsection{Materials}

All compounds were obtained in the highest-grade purity available and were stored at $0^{\circ} \mathrm{C}$ to prevent decomposition. MeSH and EtSH were supplied by Fluka (Buchs, Switzerland). n-PrSH, n-ButSH, and n-PenSH were supplied by Aldrich (Steinheim, Germany). HexSH was supplied by Lancaster Synthesis (Mu"lheim am Main, Germany).

Doubly distilled and deionized water (DDW) with a specific resistivity of $18 \mathrm{M} \Omega \mathrm{cm}$, from a Milli-Q water purification system (Millipore, Bedford, MA) was used. Spiked water samples of mercaptan solutions in 1-1000 ng/L were prepared. The samples of produced water were collected from the drainage stream in the Salman Platform located in Persian (Arabian) Gulf.

\subsection{Instrumentation}

The extractions and injections were performed by microsyringe (Agilent, CA, USA) bearing an angled cut needle tip (needle id: $0.11 \mathrm{~mm}$ and glass barrel id: $0.6 \mathrm{~mm}$ ). Gas chromatograph (PerkinElmer Instruments, Norwalk, USA) equipped with a flame-ionization detector (FID) and a split/splitless injector at $290^{\circ} \mathrm{C}$ operated at $320^{\circ} \mathrm{C}$. Helium 5.0 grade (Westfalen AG, Münster, Germany) was used as a carrier gas at $140 \mathrm{kPa}$ (inlet pressure).

In comparison to flame photometric detector (FPD), FID not only is less selective for sulfur compounds but also detects the interference of nonsulfur-bearing compounds. However, owing to availability of FID in most laboratories, it was selected as the detector of interest.

The fused silica capillary column (DB-Wax, PEG, $30 \mathrm{~m} \times 0.25 \mathrm{~mm}$ I.D., film thickness $0.5 \mu \mathrm{m}$ ) was purchased from J\&W Scientific (Folsom, CA, USA) and 
was connected to the outlet splitter (S.G.E., Melbourne, Australia). The oven temperature was programmed from $50^{\circ} \mathrm{C}(1 \mathrm{~min}$ hold $)$ at $6^{\circ} \mathrm{C} / \mathrm{min}$ to $250^{\circ} \mathrm{C}(60$ min hold). The chromatographic data were processed with TotalChrom version 6.4 software (Perkin Elmer Instruments).

\subsection{Preconcentration}

$5.0 \mathrm{~mL}$ standard solutions or real samples were placed into a $10-\mathrm{mL}$ screw-cap glass centrifuge tube with conic bottom. $0.8 \mathrm{~mL}$ of acetonitrile (as dispersive solvent) containing $10.0 \mu \mathrm{L}$ carbon tetrachloride (as extraction solvent) was rapidly transferred into the above-mentioned centrifuge tube and was gently shaken. The volume of the settled phase was determined using a $20.0 \mu \mathrm{L}$ microsyringe, and was completely transferred to another tube. After evaporation of the water under a gentle nitrogen flow, the residue was used in the next step.

\section{RESULTS AND DISCUSSION}

The optimum conditions for dispersive liquid-liquid microextraction, chromatographic separation, identification and quantification of short chain $\mathrm{n}$-alkyl $\mathrm{C}_{1}-\mathrm{C}_{6}$ mercaptans are assessed. The effects of other parameters (that influence the performance of microextraction) were investigated including the concentration and the volume of the extraction solvents as well as the dispersive solvents, the extraction and centrifugation times, $\mathrm{pH}$, temperature, and ionic strength. They were investigated and optimized in order to achieve the higher enrichment factor and recovery of short chain n-alkyl $\mathrm{C}_{1}-\mathrm{C}_{6}$ mercaptans from the samples of produced water.

\subsection{Volume of extraction solvent}

By maximizing the extraction efficiency and minimizing the phase ratio, the method is improved and the preconcentration factor is increased. It is obvious that by increasing the volume of the extraction solvent, the volume of settled phase increases. In this regard, $1.0 \mathrm{~mL}$ volumes of acetonitrile containing different volumes of carbon tetra chloride, in the range of 10-20 $\mu \mathrm{L}$, were used. The results revealed that the peak area of the analytes was maximum using $10 \mu \mathrm{L}$ of carbon tetra chloride. Increasing the volume of extraction solvent led to a decrease in the magnitude of the peak area of the analytes. This is owing to the increase in the settled volume and the consequent further decrease in the preconcentration factor. Therefore, $10 \mu \mathrm{L}$ of carbon tetra chloride was selected as the optimum value for the volume of extraction solvent in order to achieve the highest possible extraction efficiency and hence the highest preconcentration factor.

\subsection{Volume of dispersive solvent}

The volume of acetonitrile (as dispersive solvent) governs the settled volume of extraction solvent. To achieve a constant volume of the settled phase, the volume of extraction and dispersive solvents were changed simultaneously. Therefore, different volumes of acetonitrile in the range of $0.20-1.2 \mathrm{~mL}$ containing the proper volumes of carbon tetra chloride were assessed. The extractions were improved with the increase of dispersive solvent volume up to $0.8 \mathrm{~mL}$. At low volumes of acetonitrile, the cloudy state was not formed well; hence, the recovery was low. At higher volumes of acetonitrile, the extraction efficiency was reduced since the solubility of amines in water was increased. Therefore, $0.8 \mathrm{~mL}$ volume of acetonitrile was used as the optimum value for further experiments.

\subsection{Type of extraction solvent}

Selection of proper extraction solvents is the most important parameter in the optimization of the dispersive liquid-liquid microextraction. The extraction solvent should meet the below-mentioned requirements including: to be separated from analyte peaks in the chromatogram, to extract analytes well, to have larger density than sample solution (to be settled at the bottom of tube), and to form a cloudy solution of tiny droplets in the presence of dispersive solvents. Owing to high density of halogenated hydrocarbons, they were selected as extraction phases and compared for extraction of short chain $\mathrm{n}$-alkyl $\mathrm{C}_{1}-\mathrm{C}_{6}$ mercaptans from water solution. The selected extraction phases were including carbon tetrachloride $(1.59 \mathrm{~g} / \mathrm{mL})$, chloroform $(1.48 \mathrm{~g} / \mathrm{mL})$, chlorobenzene (density $1.11 \mathrm{~g} / \mathrm{mL})$, and tetrachloroethylene $(1.62 \mathrm{~g} / \mathrm{mL})$.

A series of sample solutions were studied by using $0.8 \mathrm{~mL}$ of acetonitrile (in the optimized value) containing $10 \mu \mathrm{L}$ volume of extraction solvents to achieve about $10 \mu \mathrm{L}$ volume of settled phase. To obtain the required volume of settled phase (considering the solubility of extracting solvent in aqueous phase in the presence of dispersive solvent), 20,15, 40, and $10 \mu \mathrm{L}$ of carbon tetrachloride, chlorobenzene, chloroform and tetrachloroethylene were used, respectively. The results showed that the extraction recoveries were increased with the order of carbon tetrachloride $>$ chlorobenzene $>$ tetrachloroethylene $>$ chloroform. Therefore, carbon tetrachloride was selected as the extraction solvent for the experiments.

\subsection{Type of dispersive solvent}

Selection of dispersive solvent is limited to miscible solvents with both aqueous (sample solution) and organic (extraction solvent) phases. Therefore, acetonitrile, acetone and methanol were used for this purpose. A series of sample solutions was assessed by using $0.8 \mathrm{~mL}$ of each dispersive solvents containing $10.0 \mu \mathrm{L}$ carbon tetrachloride as extraction solvent in the optimum volume. Base upon the results, the extraction efficiencies were increased as methanol $>$ acetonitrile $>$ acetone. However, owing to the better chromatographic behavior of acetonitrile with respect to methanol, acetonitrile was selected as the dispersive solvent.

\subsection{Time of extraction}

The extraction time means the time from the moment that the solution containing completely dispersed phase forms to the set interval. The longer the extraction time, the easier to reach the extraction balance. Hence, the best extraction performance was obtained. Therefore, extraction time puts an important role in the process of microextraction. In the rest of studies, a series of experiments were conducted to assess the best extraction time. Base upon the results, the extraction time of $2 \mathrm{~min}$ was the best interval to make the short chain n-alkyl $\mathrm{C}_{1}-\mathrm{C}_{6}$ mercaptans to be well extracted. In the initial $2 \mathrm{~min}$, the peak areas gradually increased with the time change because the amount of settled phase increased with the time and only a very few phase were not separated from water phase due to the solubility. However, the peak areas would decrease when the extraction time exceeded more. It was maybe owing to the volatile lost of the analytes. Finally, 2 min was selected for use.

\subsection{Selection of centrifugation time}

Centrifugation is known as an important step to achieve the best separations in dispersive liquid-liquid microextraction. The interval of centrifugation seriously affected the separation extent of the mixture and hence, sequentially affected the extraction efficiency. Commonly, a higher rate of centrifugation leads to a shorter centrifugation time. Hence, the maximum rate of $3000 \mathrm{rpm}$ was used in the experiments. In order to reach the best enrichment, centrifugation time was considered in the range of $1-5 \mathrm{~min}$. The results revealed that the peak areas gradually increased along with the prolongation of centrifugation time. When the centrifugation time was over 2 min, the peak areas almost had no further increase. Because of saving time, the interval of $2 \mathrm{~min}$ was selected as the optimal centrifugation time.

\subsection{Effect of sample pH}

Mercaptans are present as different states in the different $\mathrm{pH}$ environment. Hence, the sample enrichment is related directly to the present state of short chain $\mathrm{n}$-alkyl $\mathrm{C}_{-}-\mathrm{C}_{6}$ mercaptans in most cases. In this method, it is obvious that the $\mathrm{pH}$ of sample solutions has an important impact on the preconcentration. Therefore, the effect of sample $\mathrm{pH}$ was optimized over the range of 3-9. It was found that the extraction performance all reached a better level at $\mathrm{pH} 4-5$. When the sample $\mathrm{pH}$ was higher or lower than this interval, the peak areas decreased rapidly. In the low and high $\mathrm{pH}$ solutions, the short chain n-alkyl $\mathrm{C}_{-}$$\mathrm{C}_{6}$ mercaptans form in the cationic and anionic states, respectively. Therefore, their extraction from water to organic phase is limited. Based on thorough consideration, $\mathrm{pH} 5$ was used for further tests.

\subsection{Effect of temperature}

The temperature is one of the key parameters to carry out the dispersed phases into the sample solution completely and the analytes have the best chance and more contact area to migrate into the organic phase and realize the constructed microextraction. Therefore, it was studied in detail in the range of $40-70^{\circ} \mathrm{C}$. It was found that $70^{\circ} \mathrm{C}$ was reasonable for obtaining the best extraction. The lower recoveries at lower temperatures were owing to the poor performance of dispersing agent as well as the low diffused rate of the analytes at low temperatures. Those resulted in relatively few amount of analytes migrating into dispersed phase and led to the results of low recoveries. Furthermore, the slight decrease of recoveries of short chain n-alkyl $\mathrm{C}_{1}-\mathrm{C}_{6}$ mercaptans over $70^{\circ} \mathrm{C}$ may be owing to the loss of them (volatilization of analytes) at high temperatures. Therefore, $70^{\circ} \mathrm{C}$ was finally adopted for further experiments.

\subsection{Effect of Ionic strength}

Salting in/out is often an impact factor in the microextraction and the 
enrichment performance. Commonly, the effect of ionic strength is surveyed by adding a proper salt. In the rest of experiments, the ionic strength was optimized in the range of $2-10 \mathrm{w} / \mathrm{v} \% \mathrm{NaCl}$. The results revealed that the peak areas of analytes increased along with the increase of salt concentration. However, when the salt concentration continuously increased, the peak areas began to decrease. Hence, the salt was not added in the followed experiments.

\subsection{Analytical performance}

Using $5 \mathrm{~mL}$ of the standard solutions, the calibration curves were obtained and exhibited good linearity in a wide range of concentrations. For this aim, each standard sample was extracted by the recommended DLLME procedure at optimum conditions, and the calibration curves were obtained by plotting the peak area of each mercaptans $v s$. its initial concentration in the aqueous phase. A typical chromatogram representing the short chain n-alkyl $C_{1}-C_{6}$ mercaptans in the standard solutions is depicted in Figure 2.



Figure 2. Gas Chromatograms of standard solutions containing minimum detectable concentration of short chain n-alkyl $\mathrm{C}_{1}-\mathrm{C}_{6}$ mercaptans, respectively.

Table 1 presents the figures of merit of the proposed approach.

Table 1. Figures of merit for DLLME-GC-FID determination of six short chain n-alkyl $\mathrm{C}_{1}-\mathrm{C}_{6}$ mercaptans.

\begin{tabular}{|c|c|c|c|c|c|}
\hline $\begin{array}{c}\text { Ana- } \\
\text { lyte }\end{array}$ & $\begin{array}{c}\text { LOD } \\
(\mathrm{ng} / \mathrm{L})\end{array}$ & $\begin{array}{c}\text { Dynamic } \\
\text { Linear } \\
\text { Range } \\
(\mathrm{ng} / \mathrm{L})\end{array}$ & $\begin{array}{c}\text { Precision } \\
(\%, \mathrm{n} \\
=6)\end{array}$ & $\begin{array}{c}\text { Precon- } \\
\text { centration } \\
\text { Factor }\end{array}$ & $\begin{array}{c}\text { Extrac- } \\
\text { tion } \\
\text { Recovery } \\
(\%)\end{array}$ \\
\hline MeSH & 2.0 & $4.0-100.0$ & 6.8 & 864 & 88.1 \\
\hline EtSH & 4.0 & $6.0-80.0$ & 11.0 & 798 & 92.2 \\
\hline PrSH & 1.0 & $2.0-90.0$ & 2.6 & 830 & 86.8 \\
\hline ButSH & 2.0 & $5.0-120.0$ & 4.4 & 813 & 78.9 \\
\hline PenSH & 1.0 & $3.0-80.0$ & 9.2 & 842 & 82.4 \\
\hline HexSH & 1.0 & $2.0-110.0$ & 6.5 & 833 & 86.7 \\
\hline
\end{tabular}

The preconcentration factor was calculated as eq. 1 .

$$
P F=\frac{C_{S E T}}{C_{0}}
$$

Where $P F, C_{S E T}$ and $C_{0}$ are the preconcentration factor, the final concentration of analyte in the settled phase and initial concentration of analyte in the aqueous sample, respectively. $C_{S F T}$ was calculated by direct injection of standard solution of above-mentioned mercaptans into the GC-FID. The limits of detection (LODs) were calculated as the concentration equivalent to three times of the blank standard deviation divided by the slope of the calibration curve.

The figures of merits for above-mentioned mercaptans were determined. The linear ranges and related precisions were determined to be $4.0-100.0 \mathrm{ng} / \mathrm{L}$ $(\mathrm{n}=6, \mathrm{RSD}=6.8 \%), 6.0-80.0 \mathrm{ng} / \mathrm{L}(\mathrm{n}=6, \mathrm{RSD}=11.0 \%), 2.0-90.0 \mathrm{ng} / \mathrm{L}(\mathrm{n}$ $=6, \mathrm{RSD}=2.6 \%), 5.0-120.0 \mathrm{ng} / \mathrm{L}(\mathrm{n}=6, \mathrm{RSD}=4.4 \%), 3.0-80.0 \mathrm{ng} / \mathrm{L}(\mathrm{n}=6$, $\mathrm{RSD}=9.2 \%)$ and $2.0-110.0 \mathrm{ng} / \mathrm{L}(\mathrm{n}=6, \mathrm{RSD}=6.5 \%)$, respectively. The LODs were obtained $2.0,4.0,1.0,2.0,1.0$ and $1.0 \mathrm{ng} / \mathrm{L}$, respectively.

The extraction recovery was obtained by eq. 2 .

$$
R_{E}=\frac{C_{S E T}}{C_{0}} \cdot \frac{V_{S E T}}{V_{\Phi}} \times 100
$$

Where $E_{R} \%, V_{S E T}$ and $V_{A Q}$ are the extraction recovery, volumes of the settled phase and aqueous sample, respectively. In optimum conditions, the preconcentration factors and recovery values were obtained in the range of $798-864 \%$. The relative standard deviations (RSDs\%) at minimum detectable concentrations of above-mentioned mercaptans were found to be less than $6.7 \%$.

\subsection{Microextraction of Real Samples}

The matrix effects on the extraction were also evaluated by studding the applicability of this approach to determine the concentration of short chain $\mathrm{n}$-alkyl $\mathrm{C}_{1}-\mathrm{C}_{6}$ mercaptans in the samples of produced water taken from one of the Iranian offshore fields. The samples were extracted using DLLME method and analyzed by GC-FID. The samples were spiked with standard solutions at different concentration levels to investigate the matrix effects. The chromatogram representing the short chain n-alkyl $\mathrm{C}_{1}-\mathrm{C}_{6}$ mercaptans in the real sample of produced water is presented in Figure 3.

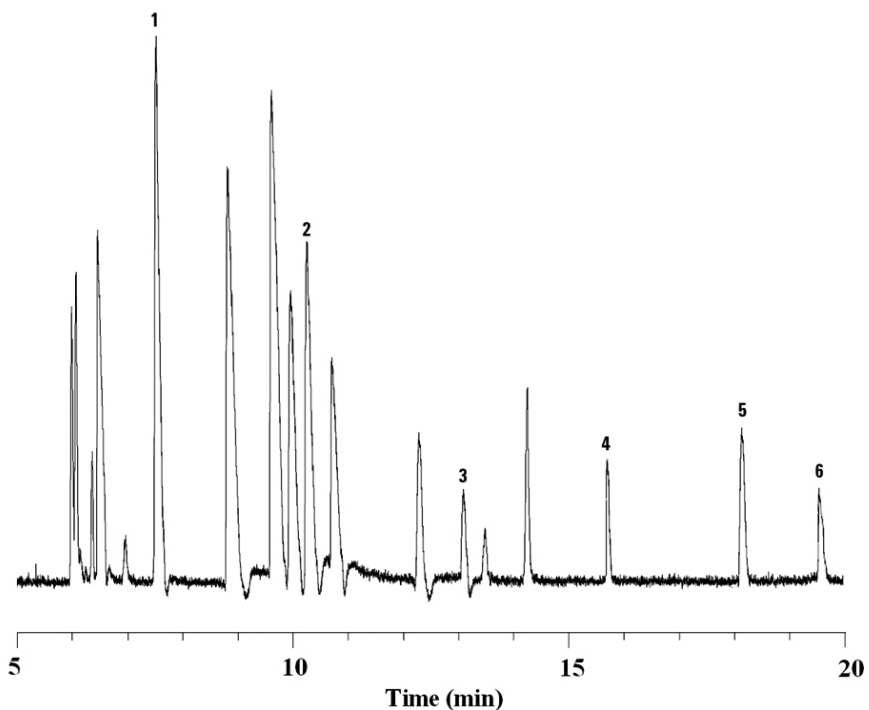

Figure 3. Gas Chromatogram of extracted n-alkyl $\mathrm{C}_{1}-\mathrm{C}_{6}$ mercaptans from produced water.

The results of relative recovery of produced water samples are shown in Table 2. Relative recoveries for all short chain $n$-alkyl $\mathrm{C}_{1}-\mathrm{C}_{6}$ mercaptans in produced water were between $108-149 \%$. These results demonstrate that the produced water matrice, in our present context, had little effect on DLLME.

Table 2. Relative recoveries and standard deviations of six short chain n-alkyl C $-C_{6}$ mercaptans from spiked produced water samples

\begin{tabular}{|c|c|c|c|}
\hline Analyte & Added (ng/L) & $\begin{array}{c}\text { Found (S.D., } \mathrm{n}=6) \\
(\mathrm{ng} / \mathrm{L})\end{array}$ & $\begin{array}{c}\text { Extraction } \\
\text { Recovery }(\%)\end{array}$ \\
\hline MeSH & 10.0 & $14.9(0.08)$ & 149 \\
\hline EtSH & 10.0 & $13.1(0.06)$ & 131 \\
\hline PrSH & 10.0 & $10.8(0.05)$ & 108 \\
\hline ButSH & 10.0 & $12.0(0.05)$ & 120 \\
\hline PenSH & 10.0 & $12.3(0.04)$ & 123 \\
\hline HexSH & 10.0 & $11.6(0.02)$ & 116 \\
\hline
\end{tabular}

3.11. Matrix Effect

In quantitative analysis, real samples can be analyzed using calibration curves established in different conditions. For instance, calibration solutions can be prepared using pure analyte and diluting to the final volume with clean solvents or it is possible to use the solvent containing co-extractives from the sample matrix. When a significant matrix effect is present, these calibrations are 
not similar (there are differences between either the slopes and/or intercepts), and different analyte contents will be obtained from the same analytical signal.

Matrix effect was evaluated by performing linear regression with a 1 / concentration factor to determine the slope of the calibration curve. The slopes from the linear calibration were used to calculate the effect ratios where the calibration slope on the matrix is divided by the slope.

In order to compare matrix effect over sensitivity, the data about slopes of calibration curve was included in water and real sample media. Figure 4 depicted the matrix effect for six short chain n-alkyl $\mathrm{C}_{1}-\mathrm{C}_{6}$ mercaptans.



Figure 4. Matrix effect for short chain n-alkyl $\mathrm{C}_{1}-\mathrm{C}_{6}$ mercaptans.

Comparing the results shows that PrSH affected by the matrix dominantly. On the other hand, HexSH depicted the most effect ratio meaning it has not been much affected by the matrix.

\section{CONCLUSION}

This study introduced the approach entitled DLLME followed by GC-FID for separation, preconcentration and determination of short chain n-alkyl $\mathrm{C}_{1}-\mathrm{C}_{6}$ mercaptans in the produced water of crude oil wells. In comparison with the conventional methods of macro-extraction, in DLLME consumption of toxic organic solvents and sample is reduced. Compared to other microextraction techniques, DLLME is preferred owing to high sensitivity, ease of operation and high preconcentration factor. Low cost, simplicity of operation and high recovery are other advantages of DLLME. In the present study, DLLME showed short extraction time and high sensitivity for preconcentration of short chain n-alkyl $\mathrm{C}_{1}-\mathrm{C}_{6}$ mercaptans in the samples of produced water. Among short chain n-alkyl $\mathrm{C}_{1}-\mathrm{C}_{6}$ mercaptans, $\mathrm{PrSH}$ and $\mathrm{HexSH}$ were affected by the matrix most and less of all, respectively.

\section{ACKNOWLEDGEMENTS}

This work was supported by Islamic Azad University (Shahreza branch) and Zanjan University.

\section{REFERENCES}

1. M. Rezaee, Y. Assadi, M.R. Milani Hosseini, E. Aghaee, F. Ahmadi, S. Berijani, J Chromatogr A 1116, 1, (2006)

2. A.E. Witter, A.D. Jones, Environ Toxicol Chem 17 (1998) 2176-2184.

3. A.E. Witter, A.D. Jones, Environ Toxicol Chem 18 (1999) 1920-1926.

4. V.B. Stein, R.S. Narang, Anal Chem 54(6), 991-992 (1982).

5. K.F. Sporek, M.D. Danyi, Anal Chem 35 (1963) 956-958.

6. P.G. Hill, R.M. Smith, J Chromatogr A 872 (2000) 203-213.

7. Q. Wang, J.M. Chong, J. Pawliszyn, Flavour Fragrance J 21 (2006) 385-394.

8. J.J. Rodriguez-Bencomo, R. Schneider, J.P. Lepoutre, P. Rigou, J Chromatogr A 1216 (2009) 5640-5646.
9. M. Mestres, M.P. Marti, O. Busto, J. Guasch, J Chromatogr A 849 (1999) 293-297.

10. J. Herszage, S.E. Ebeler, Am J Enol Vitic 62 (1999) 1-8.

11. Y. Li, X.J. Zhang, C. Chen, Prog Chem 21 (2009) 2718-2725. 